ARTICULOORICINAL

\title{
Efectos psicológicos del confinamiento social por COVID-19 en la población hondureña
}

\section{Psychological effects of social confinement due to COVID-19 on the Honduran population}

Cristhian Abate-Flores ${ }^{\mathrm{a}}$, Óscar Rolando Urtecho-Osorto ${ }^{\mathrm{b}}$, Maitée Agüero ${ }^{\mathrm{c}}$

a Dirección de Investigación Científica y Comunicación, Universidad Nacional de Policía, Tegucigalpa, Honduras

b Coordinación de Edición y Publicación, Universidad Nacional de Policía, Tegucigalpa, Honduras

c Maestría en Psicología Clínica, Escuela de Ciencias Psicológicas, Facultad de Ciencias Sociales, Universidad Nacional Autónoma de Honduras, Tegucigalpa, Honduras

\section{RESUMEN}

Esta es una investigación de enfoque mixto que tiene el objetivo de analizar los efectos psicológicos del confinamiento social por COVID-19 en la población hondureña. En la parte cuantitativa se aplicaron tres escalas tipo Likert a 487 personas (que tenían entre 15 y 66 años) con un promedio de edad de 27 años, en las que se indagó sobre la satisfacción con la vida, síntomas depresivos y felicidad. Para la recolección de la información cualitativa se creó una guía de entrevista que se aplicó a seis personas (tres hombres y tres mujeres) con una edad promedio de 32 años, para indagar sobre el malestar psicológico, bienestar emocional, vínculos familiares-sociales y el malestar físico. Se encontró en la muestra cuantitativa que las mujeres experimentan más síntomas depresivos que los hombres. Las personas que tienen un nivel académico de primaria están en el rango de moderados a graves en cuanto a síntomas depresivos, y las personas solteras, divorciadas y viudas presentan síntomas depresivos en un nivel moderado. Más del $50 \%$ de la muestra ha experimentado problemas de sueño. En la parte cualitativa se logró identificar algunas emociones negativas que se están experimentando durante la cuarentena por COVID-19: miedo, impotencia, incertidumbre y nostalgia, todo esto ligado a una sensación de pérdida de control de la vida propia. Entre las preocupaciones principales están el empleo, la alimentación, salud y economía. Sin embargo, las condiciones de la cuarentena han propiciado el diálogo y la unidad familiar.

Palabras clave: COVID, felicidad, satisfacción con la vida, depresión, bienestar psicológico

\section{ABSTRACT}

This is a mixed-focus investigation that aims to analyze the psychological effects of social confinement

Autor corresponsal

$\triangle$ Christian Abate-Flores

cabate.dnep@gmail.com 
due to COVID-19 on the Honduran population. In the quantitative part, three Likert-type scales were applied to 487 people (aged between 15 and 66 years) with an average age of 27 years, in which life satisfaction, depressive symptoms and happiness were investigated. For the collection of qualitative information, an interview guide was created that was applied to six people (three men and three women) with an average age of 32 years, to inquire about psychological distress, emotional well-being, familysocial ties and physical discomfort. It was found in the quantitative sample that women experience more depressive symptoms than men. People who have an elementary school level are in the moderate to severe range of depressive symptoms, and single, divorced, and widowed people have depressive symptoms at a moderate level. More than $50 \%$ of the sample has experienced sleep problems. In the qualitative part, it was possible to identify some negative emotions that are being experienced during the COVID-19 quarantine: fear, impotence, uncertainty and nostalgia, all this linked to a feeling of loss of control of one's life. Among the main concerns are employment, food, health and economy. However, the conditions of the quarantine have fostered dialogue and family unity.

Keywords: COVID, happiness, life satisfaction, depression, psychological well-being

\section{INTRODUCCIÓN}

La propagación de la COVID-19 a todo el mundo ha sido un fenómeno complejo por las múltiples facetas de la vida que está trastocando. Estudiar este fenómeno desde una perspectiva académica social es fundamental para comprender su impacto en las personas, a nivel psicológico y de convivencia, y también para generar insumos que permitan la toma de decisiones acertadas para paliar las secuelas más profundas que el confinamiento deje sobre la sociedad.
La COVID-19, como la denominó la Organización Mundial de la Salud (OMS), es una enfermedad provocada por un virus de la familia de los coronavirus, el SARS-CoV-2. Estos virus son responsables de causar infecciones leves en la mayoría de sus contagios, $\mathrm{y}$ en menor proporción muy graves (muchas de las personas contagiadas son asintomáticas y la cantidad de personas que requieren cuidados intensivos en relación al número de infectados es bastante baja). La enfermedad puede afectar los tractos respiratorios superiores e inferiores (Ena y Wenzel 2020). El SARS-CoV-2 no ha sido el único coronavirus que se ha propagado en lo que va del siglo XXI, ya que en 2003 se identificó el síndrome respiratorio agudo severo, conocido como SARS-CoV (García et al. 2003), y en el 2011 el síndrome respiratorio de Oriente Medio, conocido como MERS-CoV (OMS 2019). Ambos virus son de naturaleza zoonótica, es decir que se transmiten de animales a personas. En el caso del SARS, se tiene evidencia de que su transmisión pudo haber sido a través de un murciélago, y el MERS probablemente se transmitió de un dromedario o camello arábigo.

Los primeros casos de COVID-19 se dieron en Wuhan, China. En esta región para el 31 de diciembre se contabilizaban 27 neumonías relacionadas con un mercado de animales marítimos y vivos de esta zona. El proceso de contagio, igual que en el caso del SARS-CoV, fue de un murciélago a una persona (Peña-Otero et al. 2020). Ya para febrero de 2020 la enfermedad se había propagado por gran parte de Europa y otros países asiáticos, y para mayo la OMS estaba catalogando a la COVID-19 como una pandemia que estaba afectando a 114 países, dejando un gran número de infectados y víctimas mortales (OMS 2020).

La letalidad de la COVD-19, en comparación con otras enfermedades, incluso infecciones relacionadas con el coronavirus, no es tan elevada (Peña-Otero 
et al. 2020; Centro de Coordinación de Alertas y Emergencias Sanitarias 2020). Sin embargo, por su rápida propagación, la falta de conocimiento de la enfermedad, la carencia de un tratamiento para la atención de enfermos y la saturación de los sistemas de salud, la mayoría de países decidió establecer, como estrategias para frenar el contagio, medidas no farmacológicas (conocidas como NPI por sus siglas en inglés) que están orientadas, en su mayoría, a las restricciones de la movilización de las personas y al cese de actividades económicas en las regiones afectadas (CEPAL 2020).

En Honduras, los primeros casos de COVID-19 se detectaron el 10 de marzo y el primer fallecimiento se produjo el 26 de ese mismo mes (Despacho de Comunicación y Estrategia Presidencial 2020). Desde entonces, el gobierno estableció, como estrategia para evitar la propagación de esta enfermedad, la restricción de la movilidad y el cese de actividades económicas en los departamentos donde se ha registrado mayor número de contagios: Cortés y Francisco Morazán.

Este tipo de medidas, aunque han ayudado a contener la propagación de la enfermedad por el distanciamiento social que conlleva el confinamiento en los hogares, han traído repercusiones económicas y sociales que impactan la salud mental, un componente muy importante en la vida de una persona, ya que es fundamental para el desarrollo pleno de cualquier individuo. Para la OMS (2004), la salud mental es un estado íntegro que incluye aspectos como el bienestar subjetivo, autonomía, competencia, dependencia intergeneracional y la habilidad para autorrealizarse intelectual y emocionalmente. También abarca aquellos mecanismos de afrontamiento al estrés que se vive en el día a día y la realización de los objetivos que ayudan al bienestar personal. La salud mental está ligada con la salud física y social.

Existe evidencia que respalda que los periodos de cuarentena deterioran la salud mental, sobre todo porque las personas sometidas a ellos están expuestas a estresores durante y después del confinamiento. Algunos de los factores que pueden deteriorar la salud mental están relacionados con la duración de la cuarentena (entre más se prolonga, peor para el estado de salud mental de las personas). A esto se suma el temor a la infección; las personas tienen miedo al contagio por su bienestar físico y el de sus familiares, especialmente por aquellos que están dentro de los grupos vulnerables. La frustración vinculada con el detrimento de la rutina habitual, la carencia de contacto social, la percepción de pérdida de control de la vida y la sensación de aislamiento son elementos que afectan también la salud mental de las personas. A estos aspectos se agrega la adquisición de suministros inadecuados (especialmente en los estratos sociales más bajos) y la desinformación que muchas personas tienen respecto al contexto en el que viven y el desarrollo de la enfermedad, lo que puede repercutir de manera negativa en el estado mental de las personas (Brooks et al. 2020).

Hay investigaciones que presentan evidencias de que personas sometidas a cuarentena por enfermedades contagiosas experimentaron más insomnio y agotamiento como efecto del aislamiento (Bai et al. 2004). Además, estudios realizados durante el brote del SARS-CoV mostraron una relación entre la situación producida por la enfermedad y altos niveles de depresión, especialmente en la población menor a 35 años y soltera. También se logró determinar que quienes se exponían de manera altruista a la enfermedad (como médicos y enfermeras) presentaban niveles más bajos de depresión en los periodos después de la cuarentena (Liu et al. 2012).

Además de lo expuesto, los periodos de cuarentena pueden alterar otras facetas mentales de las personas, como la satisfacción con la vida, la felicidad, los niveles de ansiedad y la misma salud física. Por este motivo se considera importante conocer cómo el periodo de 
aislamiento en Honduras ha incidido sobre la salud mental de las personas. Ante este planteamiento, se debe considerar que, a diferencia de otros países donde se han implementado las mismas estrategias de confinamiento, en Honduras las personas tienen otras preocupaciones, como el desempleo, la inseguridad y la pobreza, por ende, a estos factores que alteran el desarrollo pleno de la persona se debe de sumar el encierro y el temor mismo a la enfermedad.

\section{METOdOLOGÍA}

\subsection{Diseño}

Esta investigación tiene un diseño no experimental, de corte transversal. Aborda desde un enfoque mixto las consecuencias del confinamiento en algunas ciudades de Honduras. Los datos se recolectaron y analizaron en dos momentos: uno cualitativo, vinculado con la realización de entrevistas, y otro cuantitativo, para el que se aplicaron instrumentos tipo escala de Likert.

\subsection{Población y muestra}

El objetivo de la parte cuantitativa del estudio fue trabajar con la población hondureña, para lo que se calculó una muestra de 385 personas, con un nivel de confianza de $95 \%$ y un margen de error de $5 \%$. La muestra, no probabilística, alcanzó al final 485 personas (se logró encuestar a 487 personas, pero dos encuestas no reunieron las condiciones de rigor necesarias para el análisis). La media de edad muestral fue de 27 años. Los participantes del estudio provienen de los departamentos de Cortés, Yoro, Francisco Morazán, Atlántida, Colón, Comayagua, Choluteca, El Paraíso, Intibucá, La Paz, Ocotepeque, Santa Bárbara, Lempira y Olancho. Fueron seleccionados considerando que tuvieran más de 15 años (edad que permite un razonamiento claro sobre la situación), que tuvieran acceso a contestar la encuesta por Google Forms, que estuvieran dispuestos a participar en la investigación y que su departamento o lugar de residencia estuviera en situación de cuarentena y aislamiento social. Los instrumentos aplicados se orientaron al análisis de tres aspectos psicológicos: satisfacción con la vida, síntomas depresivos y felicidad.

\subsection{Instrumentos para la recolección de datos cuantitativos}

- En la medición de la satisfacción con la vida se utilizó la transcripción del instrumento de Diener, Emmons, Larsen y Griffin: Escala de Satisfacción con la Vida, conocido como SWLS por sus siglas en inglés. El test trata de evaluar el bienestar subjetivo, concentrándose en el juicio cognitivo y global que la persona hace sobre su vida (Diener et al. 1985). Los cortes utilizados para evaluar los resultados son: 5-9 (extremadamente insatisfechas), 10-14 (insatisfechas), 15-19 (ligeramente por debajo de la media), 20-24 (puntuación media), 25-29 (puntuación alta) y 30-35 (altamente satisfechas).

- La evaluación de síntomas depresivos se realizó a partir del Patient Health Questionnaire (PHQ-9). Este instrumento está basado en los criterios del DSM-IV para diagnosticar el episodio depresivo mayor (Saldivia et al. 2019). El test cuenta con 9 ítems y la intensidad de los síntomas se puede clasificar así: 0-4 (mínimo), 5-9 (leve), 10-14 (moderado), 15-19 (moderado a grave) y 20-27 (grave) (Kroenke et al. 2001).

- Otro aspecto que se tomó en cuenta fue la felicidad. Para medirla se empleó la Escala de Felicidad de Lima (EFL), en la versión de 14 ítems validada por Árraga Barrios y Sánchez Villarroel (2012). Este instrumento pretende reportar cuatro dimensiones: el sentido positivo de la vida, la satisfacción con la vida, la realización personal y la alegría de vivir (Alarcón 2006). 
- Para la recolección de información se utilizó la herramienta de Google Forms. Para el análisis de los datos se empleó el programa JASP. Para verificar la confiabilidad de los instrumentos para la recolección de datos se empleó el Alfa de Cronbach y Omega de McDonald, tratando de garantizar que tuviesen alta consistencia interna $(>0.80)$ y que los ítems tuvieran niveles de correlación aceptables respecto al resto de la escala $(r>0.30)$.

Se empleó el coeficiente de Pearson para realizar una prueba de validez convergente y discriminante a los instrumentos. En el primero de los casos se esperó que la correlación entre las medias de las escalas SWLS y EFL fuese positiva y significativa, ya que miden constructos similares, y que la correlación de estas medias con la escala PHQ-9 fuese negativa, ya que esta mide un constructo contrario al que evalúan la EFL y la SWLS. En general, para medir los efectos de la cuarentena se utilizó un tamizaje por cada escala y se realizaron pruebas de hipótesis T-Student y ANOVA.

\subsection{Instrumento para la recolección de información cualitativa}

Para la recolección de información cualitativa se empleó una muestra por conveniencia de seis personas (tres hombres y tres mujeres) con una media de edad de 32.1 años. Los integrantes de la muestra fueron seleccionados considerando que se tratara de personas mayores de 18 años, que tuvieran acceso a la plataforma virtual Zoom y que estuvieran dispuestas a conceder la entrevista. Se utilizó una guía semiestructurada compuesta por cinco preguntas que indagaban sobre cuatro categorías de análisis (Tabla 1) construidas tomando como referencia los factores propuestos por Barrera Guzmán y Flores Galaz (2015) en su Escala de Salud Mental Positiva para Adultos.

Durante la recolección de información surgieron tres categorías emergentes: (a) fe/religión, vinculada con el uso que las personas hacen de las tecnologías del yo para enfrentar la situación (Foucault 2012; Guerrero Bejarano 2016); (b) factores vinculados que generan malestar, relacionados con los medios de difusión de información (como redes sociales y noticieros) y eventos traumáticos que tienen impacto

Tabla 1 Categorías de análisis cualitativo

\section{Factor Descripción}

Malestar psicológico Elementos cognitivos, vinculados con la personalidad y lo social, que dificultan el o pensamientos funcionamiento de la persona.

perturbadores

Bienestar emocional Emociones displacenteras que se experimentan por la situación de cuarentena, vinculadas con la salud emocional.

Vínculos familiares Habilidades y actitudes para la adaptación al tipo de interacción que está produciendo la y sociales cuarentena, a nivel familiar y social.

Malestar físico La percepción que la persona tiene sobre su salud, vinculada con la cuarentena y la propia COVID-19, y el efecto que esto tiene sobre su estado de ánimo y estabilidad mental.

Fuente: Elaboración propia con base en el trabajo de Barrera Guzmán y Flores Galaz (2015) 
sobre la salud mental; y por último (c) condiciones del entorno, que evalúa la percepción que las personas tienen sobre su medio ambiente y la medida en que esto las afecta. Hay que agregar que al momento de analizar la información se hizo una triangulación metodológica para comparar los datos cuantitativos con los cualitativos.

\subsection{Consideraciones éticas}

A todos los participantes, tanto en la muestra cualitativa como en la cuantitativa, se les presentó un consentimiento informado donde se les explicó en qué consiste la investigación, que no tendrían ningún beneficio económico por su participación en ella y que los datos recopilados serían parte de un estudio que se haría público, pero respetando en todo momento el anonimato de las fuentes. Todos ellos aceptaron voluntariamente participar en la investigación. En el caso de las personas menores de 18 años, se contó con su propia aprobación y la de sus padres para que fueran parte de la investigación.

\section{RESULTADOS}

La mayoría de los participantes en la muestra cuantitativa corresponden al sexo femenino (66.46 $\%)$, mientras que los hombres representan un 33.54 $\%$. En cuanto al nivel académico, la mayor parte de la muestra tiene estudios universitarios (73.08\%), sigue en orden de cantidad el nivel secundario $(18.84 \%)$ y en menor proporción el nivel de maestría (7.45\%), primaria $(0.41 \%)$ y doctorado $(0.21 \%)$. El rango de edad de los participantes es de 15-66 años, la mayoría de ellos se encuentran en los 15-19 años (19.38\%), 20-24 (30.01\%), 25-29 (19.36\%) y 30-34 (13.19\%).

En cuanto a la condición laboral, un $46.58 \%$ de los participantes están desempleados y el $42.86 \%$ de ellos tienen trabajo. Este dato adquiere una nueva dimensión a luz de la información cualitativa, pues uno de los temores que hay sobre la cuarentena es "el impacto que vaya a tener posteriormente todo esto a nivel económico, que las personas de repente puedan perder trabajos" (informante 5).

El estado civil que más se denotó fue el soltero (74.12 \%), el casado representó el $17.60 \%$, la unión libre un $5.59 \%$, los divorciados $1.86 \%$ y los viudos $0.83 \%$. El promedio de hijos fue de $1 \mathrm{y}$ la edad muestral de 27 . El $84.47 \%$ de la muestra se encuentra por debajo de los 36 años y el $82.40 \%$ es del departamento de Francisco Morazán.

\subsection{Pruebas de confiabilidad}

La prueba Alfa de Cronbach se le practicó a cada uno de los instrumentos aplicados: SWLS, PHQ-9 y EFL. La realización de la prueba de confiabilidad por separado se dio por la naturaleza dimensional de cada una de las mediciones, determinando los coeficientes del Alfa de Cronbach y Omega de McDonald.

Para los tres instrumentos aplicados se determinó que los ítems presentan una elevada consistencia interna (SWLS: $\alpha=0.835, \omega=842$; PHQ-9: $\alpha=0.872$, $\omega=0.875$; y EFL: $\alpha=0.915, \omega=0.917$ ). En el caso del EFL se requirió hacer uso de valores inversos para los ítems F2, F11, F12 y F14. En ninguna de las pruebas se realizó depuración de ítems, ya que los valores presentados fueron los máximos y se consideraron aceptables $(\alpha>0.80 ; \omega>0.80)$ (Quero Virla 2010).

\subsection{Prueba de validez}

Para medir la validez de los constructos de los tres instrumentos se utilizó una prueba de correlación de Pearson que analiza la convergencia y divergencia entre las medias de los resultados obtenidos en los tests (Tabla 2).

La validez de constructo se estima conveniente por la correlación positiva y altamente significativa entre las medias del instrumento EFL y SWLS ( $r=$ 
$0.784, \mathrm{p}<.001$ ), que miden dimensiones relacionadas, y negativa en las correlaciones PHQ-9 en contraste con la EFL $(r=-0.559, \mathrm{p}<.001)$ y SWLS $(r=-0.426$, $\mathrm{p}<.001)$, pues miden constructos antagónicos o contrarios entre sí.

\subsection{Pruebas T y ANOVA para grupos independientes}

Para la escala PHQ-9, que evalúa síntomas de depresión, la muestra obtuvo una puntuación valorada como moderada $(\bar{X}=10.219)$. De esta, las mujeres $(\bar{X}=10.891)$ obtuvieron una media superior a la de los hombres $(\bar{X}=8.902)$, siendo esta diferencia significativa ( $T$ de Student, $p<.05$ ). En cuanto a la condición laboral, los datos demuestran que las medias difieren en relación a esta variable, esto a partir de una prueba ANOVA $(\mathrm{p}=<.05)$, donde los arrendadores $\mathrm{y}$ desempleados se encuentran en una calificación moderada $(\bar{X}=12.900 ; 11.747)$ y los comerciantes y personas que tienen empleos en una calificación leve $(\bar{X}=9.390 ; 8.608)$. En cuanto a la edad, se halló una correlación negativa muy débil $(\mathrm{r}=-.189$, $\mathrm{p}<.001)$. En relación al nivel académico, las medias son significativamente diferentes, por la prueba de $\operatorname{ANOVA}(\mathrm{p}<.05)$, donde los que tienen solamente nivel académico primario se califican de moderadamente a graves $(\bar{X}=19.500)$, mientras que el resto se califica como moderado ( $\bar{X}=10-14)$. Los estados civiles son otro aspecto en el que difieren (ANOVA, $\mathrm{p}=<.05$ ). Los divorciados, solteros y viudos presentan síntomas depresivos moderados $(\overline{\mathrm{X}}=12.667 ; 11.092 ; 10.750)$,

Tabla 2 Correlación de validez convergente y discriminante para las tres pruebas

\begin{tabular}{|c|c|c|c|c|c|c|}
\hline Prueba & Parámetro & \multicolumn{2}{|c|}{ Media EFL } & \multicolumn{2}{|c|}{ Media SWLS } & Media PHQ-9 \\
\hline \multirow[t]{4}{*}{ Media EFL } & Pearson's r & \multicolumn{5}{|l|}{ - } \\
\hline & $\mathrm{p}$-value & \multicolumn{5}{|l|}{ - } \\
\hline & Upper 95\% CI & \multicolumn{5}{|l|}{ - } \\
\hline & Lower $95 \%$ CI & \multicolumn{5}{|l|}{ - } \\
\hline \multirow[t]{4}{*}{ Media SWLS } & Pearson's r & 0.784 & \multirow[t]{4}{*}{$* * *$} & \multicolumn{3}{|l|}{ - } \\
\hline & p-value & $<.001$ & & \multicolumn{3}{|l|}{ - } \\
\hline & Upper 95\% CI & 0.816 & & \multicolumn{3}{|l|}{ - } \\
\hline & Lower $95 \% \mathrm{CI}$ & 0.747 & & \multicolumn{3}{|l|}{ - } \\
\hline \multirow[t]{4}{*}{ Media PHQ-9 } & Pearson's r & -0.559 & $* * *$ & -0.426 & $* * *$ & - \\
\hline & $\mathrm{p}$-value & \multicolumn{2}{|l|}{$<.001$} & \multicolumn{2}{|l|}{$<.001$} & - \\
\hline & Upper 95\% CI & \multicolumn{2}{|l|}{-0.495} & \multicolumn{2}{|l|}{-0.351} & - \\
\hline & Lower 95\% CI & \multicolumn{2}{|l|}{-0.618} & \multicolumn{2}{|l|}{-0.496} & - \\
\hline
\end{tabular}

${ }^{*} \mathrm{p}<.05,{ }^{* *} \mathrm{p}<.01,{ }^{* * *} \mathrm{p}<.001$ 
mientras que los casados y quienes están en unión libre los presentan leves $(\bar{X}=7.460 ; 6.630)$.

Particular atención en esta escala merece el reactivo 3: "Ha tenido dificultad para quedarse o permanecer dormido(a), o ha dormido demasiado", donde el 50.3 $\%$ de los entrevistados afirma haber tenido problemas de sueño más de la mitad de los días o casi todos los días de la cuarentena. Este es un dato que se ha presentado en otros estudios y que se refuerza con los hallazgos cualitativos, tal como lo expresa el informante 5, agregando a la situación la causa por la que él cree que ocurre: "A veces no puedo dormir bien por el temor".

Para la escala de satisfacción con la vida la muestra obtuvo una calificación media de "insatisfecha" $(\bar{X}=18.056)$. En esta escala no hay diferencias por sexo ( $\mathrm{T}$ de Student $\mathrm{p}=0.859$ ), las medias para mujeres $(\bar{X}=18.081)$ y hombres $(\bar{X}=18.006)$ son muy similares. En cuanto al estado civil, existen diferencias entre las medias (ANOVA, $\mathrm{P}<.05$ ): los casados, divorciados, solteros y en unión libre se catalogan como por debajo de la media ( $\bar{X}=19.839 ; 15.111 ; 17.751 ; 18.000)$, mientras que quienes están en la categoría de viudos se evidencian como insatisfechos $(\bar{X}=13.500)$. En cuanto a la condición laboral, existen diferencias entre medias (ANOVA, $\mathrm{p}<.05$ ), pero cuatro categorías están calificadas por debajo de la media (arrendador, $\bar{X}=17.100$; comerciante, $\bar{X}=19.341$; desempleado, $\bar{X}=17.227$ y empleado, $\bar{X}=18.742$ ). En la edad se encontró una correlación muy débil positiva $(\mathrm{r}=0.061$, $\mathrm{p}=0.178$ ). En el nivel académico, las medias difieren (ANOVA, $\mathrm{P}<.05$ ), pero todas las categorías se encuentran bajo la media empleada en el instrumento (doctorado, $\bar{X}=18.000 ;$ maestría, $\bar{X}=19.568$; primaria, $\bar{X}=16.000$; secundaría, $\bar{X}=17.022$ y universitario, $\bar{X}$ $=4.258$ ).

En la escala de Felicidad de Lima, al hacer el análisis por sexo, se encontró que no existe diferencia (T-Student, $\mathrm{p}>$.05) pues las medias son similares en hombres $(\bar{X}=3.769)$ y mujeres $(\bar{X}=3.785)$. En la edad, la correlación de medias fue débil y positiva $(\mathrm{r}=0.127, \mathrm{p}<0.05)$. En relación con la condición laboral, existen diferencias en cuanto a las medias (ANOVA, $\mathrm{P}<0.05$ ): en este caso, los comerciantes tienen una media de 3.621, los empleados de 3.937, los arrendadores de 3.621 y los desempleados de 3.611. La media de la EFL difiere según el estado civil (ANOVA, $\mathrm{P}<0.05$ ): los casados ostentan la media más alta $(\bar{X}=4.143)$, mientras que las de los divorciados, solteros y en unión libre se asemejan $(\bar{X}=3.452$; $3.702 ; 3.886)$, pero en la categoría viudo, la media difiere totalmente del resto $(\bar{X}=2,893)$. Otra variable que difiere en sus medias (ANOVA, $\mathrm{P}<0.05$ ) es la de nivel académico (doctorado, $\bar{X}=4.143$; maestría, $\bar{X}=4.154$; primaria, $\bar{X}=2.893$; secundaria, $\bar{X}=3.575$ y universitarios, $\bar{X}=3.797$ ).

\subsection{Análisis cualitativo}

\subsubsection{Bienestar emocional}

En las entrevistas realizadas se identificó que algunas de las emociones negativas, dañinas para el bienestar psicológico, que experimentan las personas durante la cuarentena por COVID-19 son: miedo, ansiedad, nostalgia, inquietud, incertidumbre, desesperación e impotencia. En este sentido, las declaraciones del informante 1 (mujer, 21 años, licenciada en Comunicación y Publicidad) ponen en evidencia la experiencia con algunas de estas emociones:

"Eso (las decisiones del gobierno) puede hacer entrar un poco de ansiedad en las personas, pero yo creo que, aunque uno vea ciertas decisiones con las que no está de acuerdo, si uno se queda en casa, creo que corre menos riesgo. Pero sí siempre está el riesgo de la comida, aunque consigamos comida, uno no sabe si con las bolsas que trae hay contagio o algo, entonces las emociones 
pueden estar a flor de piel. (...) Hay momentos que extrañamos salir de casa, o ir a comer a un restaurante, o pasar un momento, no solamente acá; pero son cosas momentáneas, pensamos... y que no nos quitan el sueño. (...) Hay momentos en que uno pone las noticias y le llegan tantos mensajes y eso quiere como incomodarlo sentimentalmente, como revolverle la inseguridad, el miedo, el temor".

En estas declaraciones se evidencia un sentimiento de nostalgia relacionado con la imposibilidad de visitar los lugares que solían frecuentarse antes de la cuarentena. Además, hay una sensación de incomodidad vital que se vincula con la información que se recibe a través de las noticias y de mensajes vía redes sociales. Particular atención merece la ansiedad que genera la falta de confianza en las decisiones del Gobierno, y que esta ansiedad también se relaciona con el miedo al contagio por medios tan importantes como la forma en que se reciben los alimentos. Como telón de fondo de toda esta experiencia emocional negativa está la falta de control sobre los sucesos y la situación, tal como lo evidencia el informante 1 :

"La gente siente desesperación. (...) Yo creo que sí hay gente que está pasando cuestiones mentales muy fuertes a través de esto, porque es un proceso que representa mucha incertidumbre, que el humano no lo puede controlar, el Gobierno no lo puede controlar, el sistema de salud no lo puede controlar. Entonces el humano siempre quiere tener el control, entonces al verse en esta situación es un poquito complicado".

\subsubsection{Malestar psicológico o pensamientos perturbadores}

Entre las preocupaciones o pensamientos más perturbadores que tienen las personas, vinculados con la cuarentena y la pandemia de COVID-19, están los relacionados con la alimentación, la salud de los familiares, el dinero, la pérdida del empleo, la economía en general, la capacidad del sistema de salud, las decisiones del gobierno y la duración de la cuarentena. Estos pensamientos perturbadores, desagregados en esta presentación, en la práctica conforman una red de situaciones indisoluble en la que cada cosa está ligada a otra. Por ejemplo, la preocupación por los alimentos es una cuestión personal, del hogar, pero también incluye a la familia extendida que vive en una casa diferente; unido a esto está la preocupación por la salud de todo el grupo familiar y el acceso a recursos de cada uno de sus miembros. Esto puede identificarse en las declaraciones del informante 2 (hombre, 25 años, bachiller en Contaduría y Finanzas):

"Realmente preocuparme pues pensaría por mi familia, en el caso mío pues mi persona, lo que estamos viviendo en la cuarentena por las cuestiones de trabajo o empleo, dinero, comida y todo lo que se viene en sí. Vivimos en un país donde si usted no trabaja no hay salario y todos dependemos del salario. Necesitamos del dinero para poder comer, entonces en esa cuestión, en ese punto uno se preocupa por el dinero, por el salario que recibe mensualmente, ya que depende de eso".

El trabajo, además de ser una preocupación como recurso indispensable para la manutención familiar, en el contexto de la cuarentena puede jugar un rol terapéutico, tranquilizador e importante. Así lo expresa el informante 3 (mujer, 33 años, máster en Administración de Empresas):

"Fíjese que no me he sentido como desesperada o tampoco aburrida. Como tengo asignaciones propias de mi trabajo, eso me ha permitido distraerme un poco mentalmente, estar ocupada en otras cosas".

Además de lo expuesto, hay ciertas ideas obsesivas que tienen un impacto significativo en la vida diaria 
durante la cuarentena. Estas ideas se relacionan con la limpieza como medio para evitar el contagio. La informante 1 lo expresa así:

"Ella tiene niños, dice que a los niños los está metiendo en agua y jabón cada 30 minutos y está viviendo en una psicosis muy extrema".

\subsubsection{Vínculos familiares y sociales}

Es interesante lo que reflejan las entrevistas en cuanto a la vida en familia de los informantes: parece que las condiciones de la cuarentena han propiciado el diálogo y la unidad familiar. Esto lo expresan los informantes 1 y 3 :

"Actualmente somos una familia unida, tratamos de conversar, no conversamos tanto sino con casos así, o problemas que son un poco graves, somos unidos como familia y tratamos de mantenernos en casa". (informante 1)

"Esto nos ha dado la oportunidad también de hablar de otras cosas que a veces en el día a día, por el trabajo, uno ya tiene una rutina, una cierta monotonía, y el estar acá nos permite compartir otras cosas, temas ya más personales o planes". (informante 3 )

En el caso de las relaciones sociales, que tienen que ver con los vínculos de amistad, los informantes declaran que se mantienen en la normalidad gracias a las redes sociales, aunque siempre se señalan las restricciones de movilidad imperantes. Así lo expone el informante 3:

"La verdad es que no han sufrido cambios, siempre nos mantenemos en contacto. Ahora con eso de WhatsApp, Facebook, redes sociales, siempre nos mantenemos en contacto, aunque ya no es como de repente antes que nos podíamos mirar en cualquier momento".

\subsubsection{Malestar físico}

Según la información recogida en la entrevista, los informantes sienten temor de estar contagiados de COVID-19 cada vez que experimentan algún síntoma físico, aunque este corresponda a otra enfermedad y, en ocasiones, incluso cuando es un simple estornudo. Así lo dice el informante 2:

"Con una gripe uno dice ya también estoy contagiado, entonces cuesta que se quite. Actualmente estoy saliendo de un problema respiratorio y una gripe, una infección por decirlo así, y ha costado, pero pienso yo que uno se mete en esa cuestión, por decirlo con palabras así, de que se me va a quitar y no se me quita, entonces en cierto punto afecta".

Otro aspecto interesante son los síntomas físicos que no tienen como origen una enfermedad evidente y que podrían asociarse con el estrés, como la sensación de cansancio, las perturbaciones del sueño, problemas para comer y el dolor de cabeza. En este aspecto, los informantes 1 y 2 dicen lo siguiente:

"Ayer hablaba con una persona que me dice llevo días de no dormir, me duele la cabeza, siento que me explota, no como bien, no duermo bien". (informante 1)

“(Siento) como estrés, por decirlo así, como estrés, cansancio, cuando anteriormente nada que ver". (informante 2)

\subsubsection{Factores o temas emergentes}

Uno de los temas que surgió en las entrevistas es la fe, que se percibe como un factor regulador y estabilizador dentro del contexto familiar en las condiciones de la cuarentena. Se trata de un mecanismo psíquico, similar a una tecnología del yo (Foucault 2012), que se convoca conscientemente para autocalmarse. Esto se evidencia en las declaraciones de la informante 1:

"(En estos momentos) creo que una parte fundamental para nosotros, personalmente hablando, la oración ha sido un factor clave, porque eso es lo que ha traído para nosotros paz". 
En el polo opuesto de la fe surgió otro tema: los "factores vinculados que generan o intensifican el malestar" psicológico de la población. Entre estos factores están las noticias y los mensajes que se reciben por redes sociales. El informante 1 expresa las implicaciones de esta problemática de la siguiente manera:

"Pero ese es el temor que entra cuando estamos viendo tanta noticia, cuando estamos viendo tanta cosa. Hay momentos en que uno pone las noticias y le llegan tantos mensajes y eso quiere como incomodarlo sentimentalmente, como revolverle la inseguridad, el miedo, el temor".

A lo expuesto se suma el miedo al contagio y la exclusión social, dos temas que para efectos de esta investigación han sido agrupados en la categoría "condiciones del entorno". Ambos aspectos que se viven durante la pandemia están vinculados: el miedo al contagio, al síntoma del otro (incluso un pequeño estornudo o un gesto), provoca una reacción de exclusión (según la percepción de los entrevistados) y hasta una posible estigmatización y discriminación de la persona que presenta un síntoma visible que se vincule con la COVID-19. El informante 2 dice lo siguiente sobre esto:

"Genera como incomodidad en las demás personas, ¿verdad?, por la cuestión de que les han metido, por decirlo así, miedo o temor. Porque si uno va comúnmente a la pulpería y si lo miran que uno hace cualquier gesto, la gente se detiene, ya le dicen a uno tome el metro o dos metros de distancia y todo eso, entonces, aparte de tomar la seguridad que nos han dicho, prácticamente como que nos estamos excluyendo unos de otros, así siento yo".

Esta condición del entorno está asociada con los "factores vinculados que generan malestar". Sin embargo, entre estas condiciones hostiles también hay elementos positivos, como la solidaridad vecinal que puede surgir en algunos casos. Sobre este tema declara el informante 1:

"Sí he visto muchas personas perder el control, pero creo que por eso estamos todos en este mismo círculo. Cuando yo pierdo el control, otro me puede ayudar, y cuando otro pierde el control, yo puedo ayudar".

\section{DISCUSIÓN}

Muchos de los hallazgos de este estudio coinciden con los resultados obtenidos por otros investigadores en situaciones similares. Uno de ellos es la experiencia de la pérdida de control de los sucesos y situaciones de la vida diaria, también reportada por Brooks et al. (2020), vinculada con la frustración que produce el no poder continuar con la rutina habitual. En el caso de los síntomas depresivos según estado civil, Liu et al. (2012) mostraron que se ha manifestado una relación entre la situación producida por una enfermedad como el SARS-CoV y altos niveles de depresión, sobre todo en personas menores de 35 años y solteras. En este estudio se encontró que, en el caso de la muestra hondureña, los divorciados, solteros y viudos son quienes presentan los síntomas depresivos más elevados $(\bar{X}=12.667 ; 11.092 ; 10.750)$, aunque estos no son altos sino que moderados. Es importante señalar que todas estas personas tienen en común el carecer de una pareja.

En esta investigación también se halló que existe una cantidad considerable de personas que han experimentado problemas de sueño frecuentes. Esto está refrendado por la muestra cuantitativa (50.3\%) y la información cualitativa. Coincide, además, con los reportes de investigación presentados por Bai et al. (2004). Es también relevante el hallazgo de que algunas personas se sienten excluidas y hasta 
discriminadas por otras que sospechan que portan el virus, incluso cuando la única manifestación de esto es una tos o un estornudo. Este nuevo tipo de exclusión que trae la COVID-19, las experiencias de la gente, las emociones asociadas a ellas y la afectación psíquica que puede causarles, amerita estudios más profundos para los que esta investigación, al señalar la situación, puede convertirse en un precedente.

Es también importante considerar que esta investigación se ha centrado en la satisfacción con la vida, los síntomas depresivos, la felicidad (desde lo cuantitativo), las emociones (con algunos de sus desencadenantes) y el malestar psicológico que están experimentando las personas a consecuencia de la situación provocada por la COVID-19. Sin embargo, esto está lejos de agotar la temática y, solo en el plano psicológico, aún está pendiente investigar otras dimensiones de análisis importantes como la ansiedad, el riesgo suicidad, el afrontamiento del estrés y el impacto que el miedo y la cuarentena han tenido sobre las personas con trastornos psicológicos persistentes. Además, es necesario investigar, desde la psicología social, con el auxilio de la económica y la sociología, cómo la crisis que se vive ha afectado la convivencia en las comunidades, la adaptación de las personas y el impacto que todo esto tendrá en la forma de vivir e interactuar en el contexto particular de los hondureños.

\section{CONCLUSIÓN}

La experiencia de la pandemia de COVID-19 y sus consecuencias, como la cuarentena, confrontan a las personas con la pérdida del control de sus vidas, sus rutinas laborales y personales. Todo esto provoca que broten emociones como el miedo, la desesperación, la nostalgia por la forma de vida momentáneamente pérdida, incertidumbre e incomodidad. Todo esto está ligado a preocupaciones realistas (aunque a veces desbordadas por las noticias) sobre la salud propia y de los seres queridos, el empleo, la alimentación y en general la situación social del país. Ante esto es urgente también pensar en la salud mental de la población, no abordar la problemática de la COVID-19 como si únicamente atañera a lo físico o económico. Una forma práctica de afrontar esta situación, entre otras cosas, es generando canales digitales mediante los cuales las personas puedan consultar a especialistas para que les brinden apoyo psicológico ante cualquier crisis que pueda surgir por la situación que están viviendo. Estos medios también deberían ser generados y promovidos desde el Estado como una acción para proteger la salud integral de la población. Pero esto solo puede realizarse a partir de la investigación científica, es decir, con base en estudios empíricos sobre la realidad que está enfrentado la población.

\section{REFERENCIAS BIBLIOGRÁFICAS}

Alarcón, R. (2006). Desarrollo de una Escala Factorial para Medir la Felicidad. Revista Interamericana de Psicologíal Interamerican Journal of Psychology, 40(1), 99-106.

Árraga Barrios, M. V., \& Sánchez Villarroel, M. (2012).Validez y confiabilidad de la Escala de Felicidad de Lima en adultos mayores venezolanos. Universitas Psychologica, 11(2), 381-393.

Bai, Y., Chao-Cheng, L., Chin-Yuan, L., Chen, J.-Y., Ching-Mo, C., \& Chou, P. (2004). Survey of Stress Reactions Among Health Care Workers Involved With the SARS Outbreak. Psichiatric Service, 55(9), 1055-1057. doi: 10.1176/appi. ps.55.9.1055

Barrera Guzmán, M. L., \& Flores Galaz, M. M. (2015). Construcción de una escala de salud mental positiva para adultos en población. Revista Iberoamericana de Diagnóstico y Evaluación, 1(39), 22-33.

Brooks, S., Webster, R., Smith, L., Woodland, L., Wessely, S., Greenberg, N., \& Rubin, G. (2020). The psychological impact of quarantine and how to reduce it: rapid review of the evidence. The Lancet, 395(10227), 912-920. doi: 
$10.1016 / \mathrm{S} 0140-6736(20) 30460-8$

Centro de Coordinación de Alertas y Emergencias Sanitarias (2020). Actualización no 129. Enfermedad por el Coronavirus (COVID-19). 07.06.2020. Ministerio de Sanidad, Consumo y Bienestar Social, Gobierno de España. Disponible en: https://www.mscbs.gob.es/profesionales/ saludPublica/ccayes/alertas Actual/nCov-China/ documentos/Actualizacion_129_COVID-19.pdf

CEPAL (2020). América Latina y el Caribe ante la pandemia del COVID-19. Efectos económicos y sociales. Disponible en: https://repositorio.cepal.org/handle/11362/45337

Despacho de Comunicación y Estrategia Presidencial (2020). Coronavirus COVID-19 en Honduras. Disponible en: https://covid19honduras.org/

Diener, E., Emmons, R., Larsen, R., \& Griffin, S. (1985). The Satisfaction With Life Scale. Journal of Personality Assesment,49(1),71-75.doi:10.1207/s15327752jpa4901_13

Ena, J., \& Wenzel, R. (2020). Un nuevo coronavirus emerge. Revista Clínica Española, 220(2), 115-116. doi:10.1016/j. rce2020.01.001

Foucault, M. (2008). Tecnlogías del yo y otros textos. Introducción de Miguel Morey. Buenos Aires, Argentina: Editorial Paidós SAICF.

García, C., Maguiña, C., \& Gutierrez, R. (2003). Síndrome respiratorio agudo severo (SRAS). Revista Médica Herediana, 14(2), 89 - 93.

Guerrero Bejarano, M. A. (2016). La investigación cualitativa. INNOVA Research Journal, 1(2), 1-9. doi:10.33890/innova. v1.n2.2016.7

Kroenke, K., Spitzer, R., \& Williams, J. (2001). The PHQ9 Validity of a Brief Depression Severitu Measure. Journal of General Internal Medicine, 16(9), 606-12. doi: 10.1046/j.1525-1497.2001.016009606.x

Liu, X., Kakade, M., Fuller, C., Fan, B., Fang, Y., Kong, J., . . . Wu, P. (2012). Depression after exposure to stressful events: lessons learned from the severe acute respiratory syndrome epidemic. Comprehensive Psychiatry, 53(1), 1523. doi:10.1016/j.comppsych.2011.02.003

OMS (2004). Invertir en Salud Mental. Ginebra: Organización
Mundial de la Salud. Disponible en: https://apps.who.int/ iris/handle/10665/42897

OMS (2019). Coronavirus causante del Síndrome respiratorio de Oriente Medio (MERS-CoV). Disponible en: https://www. who.int/features/qa/mers-cov/es/

OMS (2020). Alocución de apertura del Director General de la OMS en la rueda de prensa sobre la COVID-19 celebrada el 11 de marzo de 2020: Disponible en: https://www. who.int/es/dg/speeches/detail/who-director-general-sopening-remarks-at-the-media-briefing-on-covid-19---11march-2020

Peña-Otero, D., Díaz-Pérez, D., de la Rosa-Carrillo, D., \& BelloDronda, S. (2020). ¿Preparados para el nuevo coronavirus? Archivos de Bronconeumología, 56(4), 195-196. doi: 10.1016/j.arbres.2020.02.009

Quero Virla, M. (2010). Confiabilidad y coeficiente Alpha de Cronbach. Telos, 12(2), 248-252.

Saldivia, S., Aslan, J., Cova, F., Vicente, B., Inostroza, C., \& Rincón, P. (2019). Propiedades psicométricas del PHQ9 (Patient Health Questionnaire) en centros de atención primaria de Chile. Revista Médica de Chile, 147(1), 53-60. doi: 10.4067/S0034-98872019000100053 